\title{
Observations of dwarfs in nearby voids: implications for galaxy formation and evolution
}

\author{
Simon A. Pustilnik \\ Special Astrophysical Observatory of Russian Academy of Sciences, \\ 369167, Nizhnij Arkhyz, Russia \\ email: sap@sao.ru
}

\begin{abstract}
The intermediate results of the ongoing study of deep samples of $\sim 200$ galaxies residing in nearby voids, are presented. Their properties are probed via optical spectroscopy, ugri surface photometry, and HI 21-cm line measurements, with emphasis on their evolutionary status. We derive directly the hydrogen mass $\mathrm{M}(\mathrm{HI})$, the ratio $M(\mathrm{HI}) / L_{\mathrm{B}}$ and the evolutionary parameter gas-phase $\mathrm{O} / \mathrm{H}$. Their luminosities and integrated colours are used to derive stellar mass $M_{*}$ and the second evolutionary parameter - gas mass-fraction $\left(f_{\mathrm{g}}\right)$. The colours of the outer parts, typically representative of the galaxy oldest stellar population, are used to estimate the upper limits on time since the beginning of the main SF episode. We compare properties of void galaxies with those of the similar late-type galaxies in denser environments. Most of void galaxies show smaller $\mathrm{O} / \mathrm{H}$ for their luminosity, in average by $\sim 30 \%$, indicating slower evolution. Besides, the fraction of $\sim 10 \%$ of the whole void sample or $\sim 30 \%$ of the least luminous void LSB dwarfs show the oxygen deficiency by a factor of $2-5$. The majority of this group appear very gas-rich, with $f_{\mathrm{g}} \sim(95-99) \%$, while their outer parts appear rather blue, indicating the time of onset of the main star-formation episode of less than 1-4 Gyr. Such unevolved LSBD galaxies appear not rare among the smallest void objects, but turned out practically missed to date due to the strong observational selection effects. Our results evidense for unusual evolutionary properties of the sizable fraction of void galaxies, and thus, pose the task of better modelling of dwarf galaxy formation and evolution in voids.
\end{abstract}

Keywords. galaxies: evolution, galaxies: formation, galaxies: dwarf, galaxies: abundances, galaxies: photometry, galaxies: statistics, large-scale structure of universe, radio lines: galaxies

\section{Introduction and Overview}

Studies of galaxies in low-density environments were tempting in the hope to probe the basics of galaxy evolution in isolation. The modern concepts suggest however that even the most isolated objects are related to and influenced by the baryon flows of adjacent filaments. Observational studies of galaxy samples in voids, based on large deep surveys (SDSS, 2dRGS), were mostly limited by large distant voids ( $D \sim 100-200 \mathrm{Mpc}$ ). As a consequence, they probed only the upper part of void galaxy luminosity function $\left(M_{\mathrm{r}}<-16\right)$. Only subtle or at most moderate differences with wall galaxies were found on their SFR and colours.

The complementary approach to study tens-hundred less luminous galaxies in nearby voids was suggested in Pustilnik \& Tepliakova (2011). The first void intrinisically faint galaxy sample (down to $M_{\mathrm{B}}=-11$ ) was drawn up in the nearby Lynx-Cancer void $\left(D_{\text {centre }}=18 \mathrm{Mpc}\right)$, currently including over 100 objects. Several interesting findings on void galaxy evolution (see below) evidense for importance of this direction and emphasize the need of larger statistics and detailed studies of the least luminous void galaxies. 


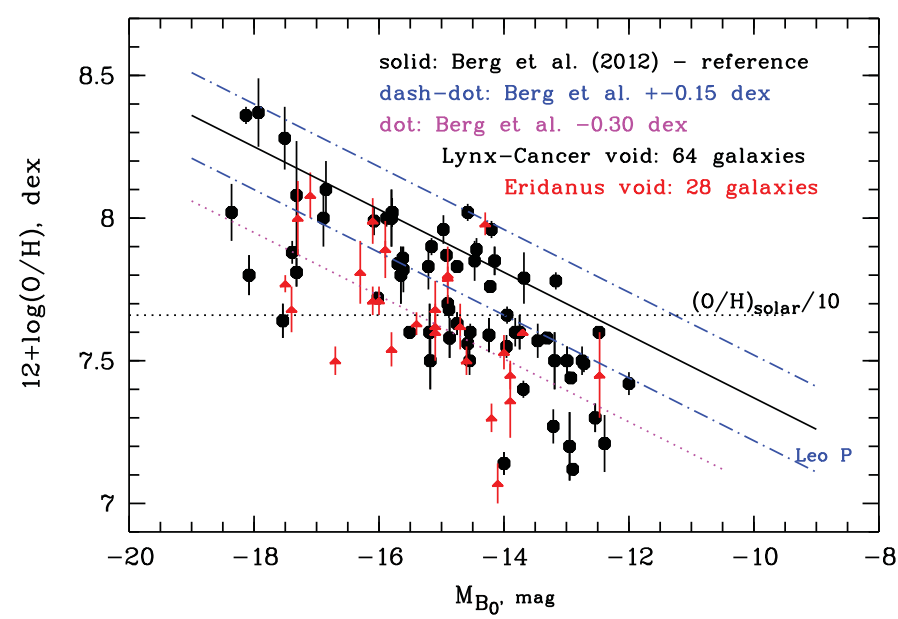

Figure 1. $\mathrm{O} / \mathrm{H}$ (with error bars) vs $M_{\mathrm{B}}$ relation for 92 galaxies in the Lynx-Cancer (filled octogons) and Eridanus (filled triangles) voids in respect of similar galaxies in denser environment (Berg et al. 2012, "reference"). The significant systematic O/H drop in void galaxies is evident as well as the sizable fraction of strong outliers (deficiency of $\mathrm{O} / \mathrm{H}$ by factor of 2-5) (Pustilnik et al. 2011, 2014; Kniazev et al. 2014, in prep.)

\section{Ongoing project and intermediate results}

To significantly encrease the number of faint void galaxies and conduct more reliable statistical study of their properties, we work on the revision of the sample of nearby voids and the sample of galaxies residing in them. In particular, galaxies residing in Monoceros and Cetus voids and in the equatorial part of Eridanus void (Fairall, 1998) are added to the current sample of the Lynx-Cancer void. Some of the important results on the evolutionary status of void galaxies are illustrated below.

In Fig. 1 we summarise determinations of gas-phase $\mathrm{O} / \mathrm{H}$ in 92 galaxies residing in the Lynx-Cancer (filled octogons) and Eridanus (filled triangles) voids (Pustilnik, Tepliakova \& Kniazev, 2011, Pustilnik et al. in prep., Kniazev et al. in prep.). In the large fraction of studied spectra no [OIII] $\lambda 4363$ line was detected, and hence semi-empirical method by Izotov \& Thuan (2007) was used to determine $\mathrm{O} / \mathrm{H}$. These $\mathrm{O} / \mathrm{H}$ are shown vs absolute blue magnitudes $M_{\mathrm{B}}$. For comparison we use the sample of similar galaxies from the Local Volume for which the confident $\mathrm{O} / \mathrm{H}$, distances and $M_{\mathrm{B}}$ are known (Berg et al., 2012). Their linear regression of $12+\log (\mathrm{O} / \mathrm{H})$ and $M_{\mathrm{B}}$ is shown by solid line, while $\pm 1 \sigma$ rms scatter in $\mathrm{O} / \mathrm{H}(0.15 \mathrm{dex})$ are shown by dash-dotted lines. The substantial shift of the whole void $\mathrm{O} / \mathrm{H}$ data below the 'reference' Berg et al. relation is well seen. Moreover, the sizable fraction of void galaxies shows the $\mathrm{O} / \mathrm{H}$ deficiency of more than by factor of two (up to five).

Another important result comes from mass photometric study of Lynx-Cancer void galaxies based on the SDSS database (Abazajian et al. 2009). In particular, we determined ugri colours of outer parts for 85 void galaxies and compared them with PEGASE2 (Fioc \& Rocca-Volmerange, 1999)) evolutionary tracks for two extreme SF laws: instantaneous and continuous with constant SFR (Fig. 2). While the great majority of void galaxies started their main star formation 7-14 Gyr ago, for about $15 \%$ of the sample we have clear indication of the retarded main star formation, commenced 1-5 Gyr ago.

The third direction in study of void galaxies is related to their HI content and structure. Integrated HI data on 96 Lynx-Cancer void galaxies (Pustilnik, Martin, in prep.) indicate that void objects are in average gas-rich, with median $M(\mathrm{HI}) / L_{\mathrm{B}}=1.2, \sim 40 \%$ higher than 


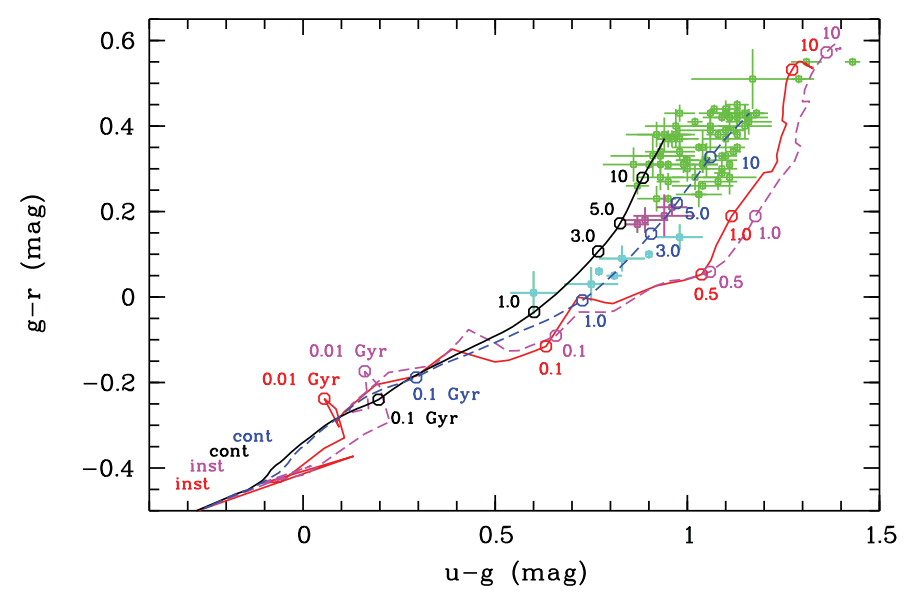

Figure 2. Age indicators: ugr colours of outer parts of 85 Lynx-Cancer void galaxies superimposed on PEGASE2 evolutionary tracks. $\sim 15 \%$ show retarded main Star Formation, started only 1-5 Gyr ago (see Perepelitsyna et al., 2014)). Solid lines correspond to Salpeter IMF, while dashed lines - to Kroupa et al. IMF.

for the sample of similar galaxies in denser environment. Mapping of their HI with Giant Meterwave Radio Telescope leads to discovery of extremely gas-rich LSB dwarfs with $M(\mathrm{HI}) / L_{\mathrm{B}}=10$ and 25 , and $M_{\text {gas }} / M_{\text {bary }}>0.99$ (Chengalur \& Pustilnik, 2013; see Fig. 3, left). Such 'unevolved' galaxies are found mostly among the least luminous void galaxies. They can be not rare among void objects with $M_{\mathrm{B}} \gtrsim-11$, but due to severe observational selection effects they escape appearance in common wide-angle spectral surveys. Another interesting result of HI-mapping of three the most isolated void LSBD galaxies shows their disturbed morphology (Fig. 3, right). Probably here we see the most clear cases of cold accretion along void filaments (Chengalur et al. in prep.).

\section{Implications}

Summarising all above and some published findings on properties of galaxies residing in nearby voids, we notice the following implications:

- void galaxies in average show slower chemical evolution, having $\mathrm{O} / \mathrm{H}$ in average $30 \%-40 \%$ lower than similar galaxies in denser environment; $\sim 10 \%$ of void galaxies have $\mathrm{O} / \mathrm{H}$ lower by $2-5$ times, indicating their unusual evolutinory status.

- ugri colours of outer parts for $\sim 15 \%$ void galaxies indicate the main SF episode started $\sim 1-5$ Gyr ago.

- More than a half of void galaxies are gas-rich, with $M(\mathrm{HI}) / L_{\mathrm{B}}>1$. Extremely gasrich dwarfs, with $M(\mathrm{HI}) / L_{\mathrm{B}}=4-25$ already found in voids can be not rare among the least luminous dwarfs $\left(M_{\mathrm{B}}>-11\right)$.

- All together these results imply that evolution of void galaxies in average goes substantially more slowly. In addition, there are indications on that $\sim 10 \%$ void galaxies formed with significant delay. This fraction reaches $\sim 30 \%$ if we consider the least luminous void LSB dwarfs.

Acknowledgements. SAP is grateful to A. Kniazev, J.-M. Martin, J. Chengalur, A. Tepliakova, Y. Perepelitsyna and E. Safonova for fruitful collaboration and their contribution to the discussed topics. The author acknowledges the partial support of this work through RFBR grant 14-02-00520 and IAU travel grant. 

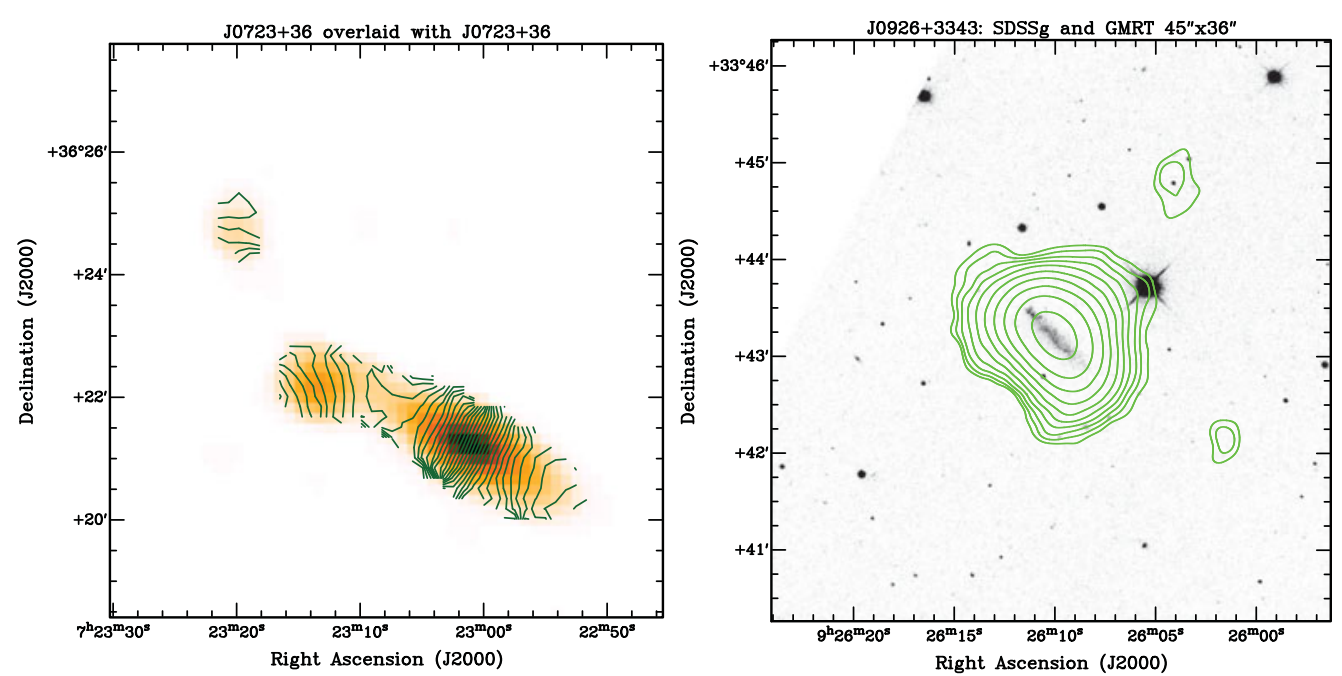

Figure 3. Left panel: Extremely gas-rich dwarf triplet J0723+36 near the centre of LynxCancer void, at $D=16 \mathrm{Mpc}$, with $M(\mathrm{HI}) / L_{\mathrm{B}}$ of $\sim 3,10$ and 25 . The two more massive members appear to experience a minor merger, while the least massive and most gas-rich dwarf at the NE is still well separated. HI column density is shown in grey scale, while contures show isovelocity lines with step of $6 \mathrm{~km} \mathrm{~s}^{-1}$. This finding can be a hint to possible hidden void population of very gas-rich low mass galaxies (Chengalur \& Pustilnik, 2013). Right panel: Disturbed HI morphology in isolated void LSBD galaxies: the 2-nd most metal-poor LSBD J0926+3343 (Chengalur et al., in prep.). HI column density (in contures) is superimposed on $g$-band SDSS image. Evidence for cold accretion?

\section{References}

Abazajian K. N., Adelman-McCarthy J. K., Agüeros M. A. et al., 2009, ApJS, 182, 543

Berg D. A., Skillman E. D., Marble A. R., et al. 2012, ApJ, 754, 98

Chengalur J. N. \& Pustilnik S. A. 2013, MNRAS 428, 1579

Fairall A., 1998, Large-Scale Structures in the Universe, Wiley-Praxis, $196 \mathrm{pp}$.

Fioc M. \& Rocca-Volmerange B. 1999, arXiv:astro-ph/9912179

Izotov Y. I. \& Thuan T. X. 2007, ApJ 665, 1115

Kreckel K., Platen E., Aragon-Calvo M. A., et al. 2012, AJ 144, 16

Perepelitsyna Y. A., Pustilnik S. A., \& Kniazev A. Y. 2014, Astroph. Bull. 69, 247 (arXiv:1408.0613)

Pustilnik S. A. \& Tepliakova A. L. 2011, MNRAS 415, 1188

Pustilnik S. A., Tepliakova A. L., Kniazev A. Y., et al. 2010, MNRAS 401, 333

Pustilnik S. A., Tepliakova A. L., \& Kniazev A. Y. 2011, Astroph. Bull. 66, 255 (arXiv:1108.4850)

Pustilnik S. A., Tepliakova A. L., \& Kniazev A. Y. 2011, MNRAS 417, 1335

Pustilnik S. A., Martin J.-M., Lyamina Y. A., \& Kniazev A. Y. 2013, MNRAS 428, 1579 